\title{
Research on Reliability of Knowledge-Updating in a Kind of Network Knowledge System
}

\author{
Jinghu Shen ${ }^{1}$, Zhe Yin ${ }^{1,2}$, Yun-fei Guo ${ }^{1}$ \\ ${ }^{1}$ Mathematics Department, Yanbian University, Jilin, China \\ ${ }^{2}$ Department of Information Management, Peking University, Beijing, China \\ E-mail: yinzhe@ybu.edu.cn \\ Received June 11, 2011; revised September 14, 2011; accepted September 22, 2011
}

\begin{abstract}
The definition of reliability of knowledge-updating in network knowledge resource management system (NKRM system for brief) has been given in this paper, which has important reference value to the evaluation of NKRM system. Besides, two kinds of models in NKRM system have been introduced. And one of the most common methods to improve reliability is the redundant technique. However, reliability and efficiency are inconsistent each other. By treating each knowledge as one part of the system, this paper proposes method of joint reserve of knowledge that may resolve this problem.
\end{abstract}

Keywords: Reliability, Redundant, Knowledge Resource Management, Joint Reserve

\section{Introduction}

Moore [1] did the classification according the functions of spare parts. Because of the influence of spare parts to manufacture and economy, many scholars have studied the amounts of the spare parts needed. P. Flint [2] provided the advice that we should develop the fellowship and the resource sharing to reduce the cycle time. Besides, Foote [3] studied stocks prediction, and Luxhoj and Rizzo [4] obtained the method of amounts of spare parts needed of the same set based on the set model. Kamath [5] used the Bayesian method to predict the amounts of spare parts needed. NKRM system has been introduced in this paper, which is shown as follows: (Figure 1).

Method of joint reserve of knowledge in NKRM system has been proposed to improve the reliability and efficiency.

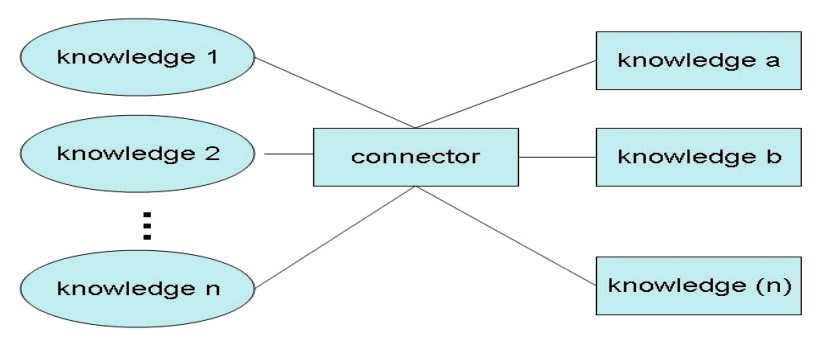

Figure 1. Knowledge resource management system.

\section{Self-Updated Model and Calculation of Its Demand of Knowledge}

\subsection{Reliability of Knowledge-Updating}

Probability that the percentage of amounts of knowledge-updating are more than a certain value, that is: $A(x)=P(X>x)$, where $x$ denotes the percentage of amounts of knowledge-updating.

Assume that reserve of knowledge of a certain new NKRM system starts from the zero moment, assessment will be done every $a(a>0)$. If the management system is normal(that is, all the knowledge are the newest), we will continue use these knowledge; otherwise, we will do self-updating to the knowledge, that is, after the temporary self-updating which cost time $b(0<b<a)$, management system recovers "normal" and proceeds reserve of knowledge, and this kind of model is called self- updated model, which is as follows: (Figure 2)

For briefly expressing, state random variable $X_{t}$ is introduced.

$$
X_{t}=\left\{\begin{array}{l}
1, \text { system is normal at time } t \\
0, \text { system fails at time } t
\end{array}\right.
$$

Let $F(t)$ be the distribution function of random variable $Z$ which is the first failure time of NKRM system. From the assumption of the model we can know, when $b=0$, availability of system at time $t$ (probability 


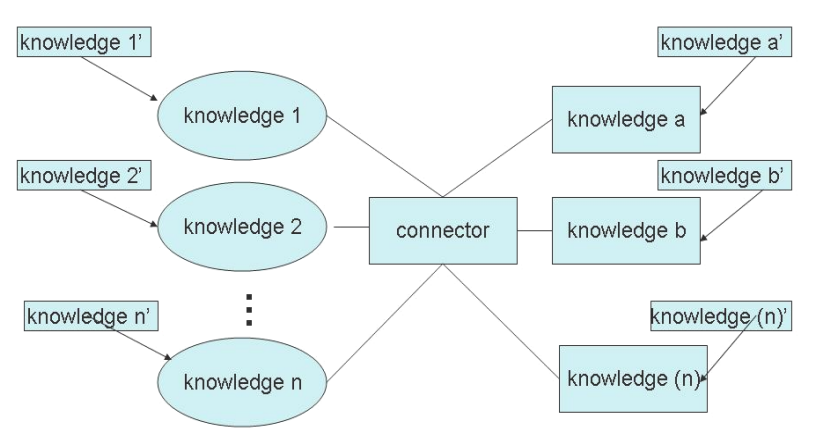

Figure 2. Self-updated model.

of system is in the normal state at time t) $A(t)=$ $P\left(X_{t}=1\right)=1-F(t)$; when $b>0$, note $a_{k}=k \cdot a$ $(k=0,1, \cdots), \quad b_{k}=k \cdot a+b(k=1,2, \cdots), \quad A(t)$ satisfies these formulas according to the model assumption: when

$$
a_{0}<t \leq a_{1}, A(t)=1-F(t)
$$

when $a_{k}<t \leq b_{k}(k>1)$

$$
A(t)=P\left(X_{t}=1 \mid X_{a k}=1\right) P\left(X_{a k}=1\right)=\frac{1-F(t)}{1-F\left(a_{k}\right)} A\left(a_{k}\right)
$$

when $b_{k}<t \leq a_{k+1}(k \geq 1)$

$$
\begin{aligned}
A(t)= & P\left(X_{t}=1, X_{a k}=0\right)+P\left(X_{t}=1, X_{a k}=1\right) \\
= & P\left(X_{t}=1 \mid X_{a k}=0\right) P\left(X_{a k}=0\right) \\
& +P\left(X_{t}=1 \mid X_{a k}=1\right) P\left(X_{a k}=1\right) \\
= & P\left(X_{t}=1 \mid X_{b k}=1\right) P\left(X_{a k}=0\right) \\
& +P\left(X_{t}=1 \mid X_{a k}=1\right) P\left(X_{a k}=1\right) \\
= & \frac{1-F(t)}{1-F\left(b_{k}\right)}\left(1-A\left(a_{k}\right)\right)+\frac{1-F(t)}{1-F\left(a_{k}\right)} A\left(a_{k}\right)
\end{aligned}
$$

From (2) and (3) we can see that $A(t)$ can be obtained once we calculate all the $A\left(a_{k}\right), k=1,2, \cdots,[t / a]^{*}$. Let $t=a_{k+1}$ in (3), when $k \geq 1$, we have:

$$
\begin{aligned}
A\left(a_{k+1}\right)= & \frac{1-F\left(a_{k+1}\right)}{1-F\left(b_{k}\right)} \\
& +\left[\frac{1-F\left(a_{k+1}\right)}{1-F\left(a_{k}\right)}-\frac{1-F\left(a_{k+1}\right)}{1-F\left(b_{k}\right)}\right] A\left(a_{k}\right)
\end{aligned}
$$

According to the formula above and $A\left(a_{1}\right)=1-F\left(a_{1}\right)$, we can calculate $A\left(a_{k}\right), k=1,2, \cdots,[t / a]^{*}$, and then $A(t)$ can be obtained.

\subsection{Calculation of Demand of Knowledge}

Suppose there are $N$ systems starting the reserve of knowledge from time $t=0$ at the same time on the same condition, besides, the state of these $N$ systems is independent each other. Then minimum of probability that there are at least $N$ normal regional sub-systems at the given moment during the reserve period is not less than $P_{0}$ is:

$$
\begin{gathered}
M=\min \left\{m ; \sum_{j=N}^{N+m}\left(\begin{array}{c}
N+m \\
j
\end{array}\right) A(t)^{j}(1-A(t))^{N+m-j}\right. \\
\left.\geq P_{0}, 0 \leq t \leq T_{0}\right\}
\end{gathered}
$$

$M$ is just the amount of the reserve of knowledge we needed.

\section{Knowledge-Introduced Model}

The basic assumption is similar with the self-updated model, the difference is that the updating process will be finished by the introducing other knowledge from elsewhere when the knowledge should be updated, which is shown as follows: (Figure 3).

\section{Calculation of Amounts of Joint Reserve of Knowledge Needed}

Generally, a knowledge updating of NKRM system in different regions is carried out independently. However, considering the problem of reducing the costs as possible as we can on the condition that the joint reserve of knowledge model is satisfied, we propose a new reserve way-joint reserve of knowledge, that is the method that amounts of joint reserve of knowledge needed are calculated uniformly after determining sum of NKRM system in each region. Assume that there are $\mathrm{n}$ systems which belongs to similar areas, and once there is a new knowledge such as knowledge 1', knowledge 2', ..., knowledge $n$, these similar systems can update the new knowledge at the same time, that is, the new knowledge can beshared in these similar systems, which is as show as Figure 4:

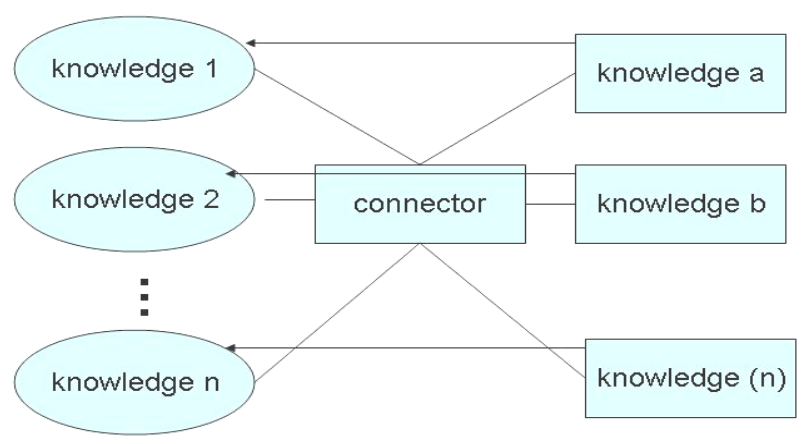

Figure 3. Knowledge-introduced model. 


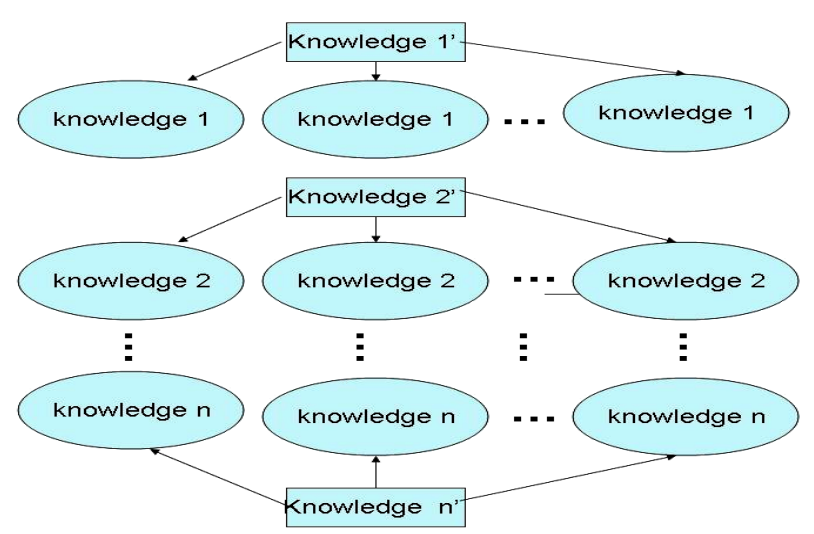

Figure4. Joint reserve of self-updated model.

In the "self-updated" model, suppose there are $N_{1}$ systems in region $\mathrm{A}$, starting the reserve of self-updating from time $t=0$ at the same time on the same condition, besides, the state of these $N_{1}$ systems is independent each other. Then minimum of probability that there are at least $N_{1}$ normal systems during the reserve period isn't less than $P_{0}$ is:

$$
\begin{gathered}
M_{1}=\min \left\{m_{1} ; \sum_{k=N_{1}}^{N_{1}+m_{1}}\left(\begin{array}{c}
N_{1}+m_{1} \\
k
\end{array}\right) A(t)^{k}(1-A(t))^{N_{1}+m_{1}-k} \geq P_{0},\right. \\
\left.0 \leq t \leq T_{0}\right\}
\end{gathered}
$$

At the same time, there are $N_{2}$ systems meeting "self-updated" model, the conditions are the same as region A, then amounts of reserve of knowledge needed $-M_{2}$ is:

$$
\begin{gathered}
M_{2}=\min \left\{m_{2} ; \sum_{k=N_{2}}^{N_{2}+m_{2}}\left(\begin{array}{c}
N_{2}+m_{2} \\
k
\end{array}\right) A(t)^{k}(1-A(t))^{N_{2}+m_{2}-k} \geq P_{0},\right. \\
\left.0 \leq t \leq T_{0}\right\}
\end{gathered}
$$

According the idea of joint reserve of knowledge, sum of these $N_{1}+N_{2}$ systems can be treated as $N$ joint reserve of NKRM systems, then amounts of knowledge needed $-M$ is:

$$
\begin{gathered}
M=\min \left\{m ; \sum_{k=N}^{N+m}\left(\begin{array}{c}
N+m \\
k
\end{array}\right) A(t)^{k}(1-A(t))^{N+m-k} \geq P_{0},\right. \\
\left.0 \leq t \leq T_{0}\right\}
\end{gathered}
$$

\section{Calculation Instances}

1) Suppose there are $10(N=10)$ same NKRM systems, which obey Weibull distribution- $w(m, \eta)$, where
Table 1. Simulation result of "self-updated" model.

\begin{tabular}{cccccccccc}
\hline$m$ & $\eta$ & $T$ & $N_{1}$ & $N_{2}$ & $N$ & $M_{1}$ & $M_{2}$ & $M$ & Confidence level 1- $\alpha$ \\
\hline 1.5 & 30.0 & 20. & 4 & 6 & 10 & 6 & 8 & 12 & 0.9 \\
1.5 & 30.0 & 10.0 & 5 & 5 & 10 & 3 & 3 & 4 & 0.9 \\
1.5 & 30.0 & 10.0 & 4 & 6 & 10 & 2 & 3 & 4 & 0.9 \\
1.0 & 25.0 & 20.0 & 5 & 5 & 10 & 11 & 11 & 19 & 0.9 \\
1.0 & 25.0 & 20.0 & 4 & 6 & 10 & 9 & 13 & 19 & 0.9 \\
\hline
\end{tabular}

Table 2. Simulation result of "knowledge-introduced" model.

$\begin{array}{llllllllll} & \eta & T & N_{1} & N_{2} & N & M_{1} & M_{2} & M & \text { Confidence level } 1-\alpha\end{array}$

\begin{tabular}{cccccccccc}
\hline 2.0 & 30.0 & 20.0 & 4 & 6 & 10 & 4 & 6 & 9 & 0.9 \\
2.0 & 25.0 & 20.0 & 5 & 5 & 10 & 8 & 8 & 14 & 0.9 \\
2.0 & 25.0 & 20.0 & 4 & 6 & 10 & 7 & 10 & 14 & 0.9 \\
1.0 & 20.0 & 10.0 & 5 & 5 & 10 & 6 & 6 & 11 & 0.9 \\
1.0 & 20.0 & 10.0 & 4 & 6 & 10 & 5 & 7 & 11 & 0.9 \\
\hline
\end{tabular}

examination time interval $a=1.0$, training time $b=0.2$, repair rate $P_{0}=0.9$ and predetermined period of reserve of knowledge $T=20.0$. In the "self-updated" model, taking some groups of parameter to simulate, results are shown as Table 1:

From Table 1 above, we can see $M<M_{1}+M_{2}$.

2) Next, simulation of "knowledge-introduced" model is also carried out on the same condition, whose results are show as Table 2 below:

From Table 2 we can also obtain that $M<M_{1}+M_{2}$.

\section{Conclusions}

Through treating each knowledge as one part of the system, this paper proposes method of joint reserve of knowledge that can improve reliability and efficiency. Besides, we can get the conclusion that amounts of knowledge needed of joint model are less than those of model in which knowledge are updated or introduced separately, which shows the importance of the knowledge-sharing.

\section{References}

[1] R. Moore, "Establishing an Inventory Management Program,” Plant Engineering, Vol. 50, No. 3, 1996, pp. 113-116.

[2] P. Flint, "Too Much of a Good Thing: Better Inventory Management Could Save the Industry Millions While Improving Reliability,” Air Transport World, Vol. 32, No. 9, 1995, pp. 103-106.

[3] B. Foote, "On the Implementation of a Control-Based Forecasting System for Air-Craft Spare Parts Procurement,” IIE Transactions, Vol. 27, No. 2, 1995, pp. 210-216. doi:10.1080/07408179508936733 
[4] J. T. Luxhoj and T. P. Rizzo, "Probabilistic Spaces Provisioning for Repairable Population Models," Journal of Business Logistics, Vol. 9, No. 1, 1988, pp. 95-117.

[5] K. R. Kamath and T. P. M. Pakkala, “A Bayesian Ap- proach to a Dynamic Inventory Model Under an Unknown Demand Distribution," Computers and Operations Research, Vol. 29, No. 4, pp. 403-422. doi:10.1016/S0305-0548(00)00075-7 